\title{
DYNAMIC FRACTURE ANALYSIS OF A FINITE CRACK SUBJECTED TO AN INCIDENT HORIZONTALLY POLARIZED SHEAR WAVE
}

\author{
YI-SHYONG ING and CHIEN-CHING MA $\dagger$ \\ Department of Mechanical Engineering, National Taiwan University, Taipei, Taiwan 10617 , \\ Republic of China
}

(Received 14 December 1995 ; in revised form 14 Mav 1996)

\begin{abstract}
The transient response for diffraction of an incident horizontally polarized shear wave by a finite crack in an unbounded elastic solid is investigated in this study. In analyzing this problem, an infinite number of diffracted waves generated by two crack tips must be taken into account that will make the analysis extremely difficult. An alternative methodology different from the conventional superposition method is used to construct the reflected and diffracted fields. The complete solutions are determined by superposition of proposed fundamental solutions in the Laplace transform domain. The fundamental solutions to be used are the problems for applying exponentially distributed (in the Laplace transform domain) traction and screw dislocation on the crack faces and along the crack tip line, respectively. The exact transient closed form solutions of dynamic stress intensity factor for two crack tips are obtained and expressed in very simple and compact formulations. Each term in the formulations has its own physical meaning. The solutions are valid for an infinite length of time and have accounted for the contributions of an infinite number of diffracted waves. Numerical results of both tips for different incident angles are evaluated which indicate that the dynamic stress intensity factors will oscillate near the correspondent static values after the first three waves have passed the specified crack tip. Some discrepancies of the numerical results compared with available solutions are discussed in detail. (c) 1997, Elsevier Science Ltd. All rights reserved.
\end{abstract}

\section{INTRODUCTION}

Recently, the transient response of a solid medium containing a crack-like flaw under dynamic loads has received much attention. Scattering of elastic waves by cracks has attracted attention over the years for its importance towards the nondestructive evaluation of materials and the dynamic fracture analysis of materials. The interaction of a stress wave with a crack is a complicated problem and the analysis is mainly restricted to relatively simple problems. Most of the work, however, has been directed towards the solution of problems with a semi-infinite crack subjected to symmetry distributed impact loading on crack faces. The complete solutions mentioned above can be obtained by integral transform methods in conjunction with direct application of the Wiener-Hopf technique (Noble, 1958) and the Cagniard-de Hoop method (de Hoop, 1958) of Laplace inversion. If the cracked problem has a characteristic length or the loading condition is unsymmetrical, then the usual procedure using integral transform methods does not apply.

The stress intensity factors at the edges of a finite crack upon diffraction of a timeharmonic wave have been obtained by Loeber and Sih (1968) and Sih and Loeber (1968; 1969). If integral transforms are applied to solve the transient response of a finite crack subjected to dynamic loading, a relationship among sectionally analytic functions will be obtained which is more complicated than the form of the standard Wiener-Hopf equations. The generalized Wiener-Hopf equation can be solved iteratively to obtain the complete transient solution, and only the first step in the iteration process has been carried out. Thau and Lu (1971), following the work of Kostrov (1964) and Flitman (1963), treated the analogous transient problem of diffraction of an arbitrary plane dilatational wave by a finite crack and a finite rigid ribbon in an infinite elastic solid from the iteration process. Their results are exact only at the time interval that the dilatational wave has travelled the length of the crack twice. Sih and Embley (1972) have studied the near field solution of a 
finite crack under transient in-plane loading. They reduced the mixed boundary value problem to a standard Fredholm integral equation and subsequently inverted the Laplace transform of the stress components by a combination of numerical means and an application of the Cagniard inversion technique. A class of problems involving interaction between a finite crack and other boundaries was considered by Chen $(1977 ; 1978)$ and Itou (1980; 1981). With the exception of Loeber and Sih who considered the time-harmonic incident wave, all of the authors mentioned above have simplified their problems by assuming the symmetry distributed loading conditions, and finally used a numerical Laplace inversion technique to obtain the solutions in the physical domain. Because of the mathematical difficulties, the close form analytical solution for the problem of a finite crack subjected to transient waves is very rare.

The problem of an unbounded medium containing a semi-infinite crack subjected to a pair of concentrated loadings on the faces of the crack has been investigated by Freund (1974). A straightforward application of the Wiener-Hopf method is not successful and the transient solution was obtained by Freund (1974) by an indirect approach based on the superposition of moving dislocations. He proposed a fundamental solution arising from an edge dislocation climbing along the line ahead of the crack tip with a constant speed to overcome these difficulties of the case with a characteristic length. The solution can be constructed by taking an integration over a climbing dislocation of different moving velocity. Basing his procedure on this method, Brock $(1982 ; 1984)$, Brock et al. (1985), and Ma and Hou $(1990 ; 1991)$ have analyzed a series of problems of a semi-infinite crack subjected to impact loading on crack faces. A thorough summary of the application of the main direct methods of analysis for transient problems in dynamic fracture for elastic or inelastic problems has been given by Freund (1990). Freund (1990) has suggested an alternate approach based on the aforementioned moving dislocation solution to examine the same finite-crack problem that had been solved by Thau and Lu (1971). In practice, however, the alternate approach provided a solution that is valid for the same time range as before.

Kostrov (1966) and Achenbach (1970a; 1970b) have used the method based on Green's function to solve the problems of crack propagation for anti-plane deformation. In their studies, the region of integration for the integral equation is in a complicated shape, generally being bounded by a hyperbola and a number of straight lines. For points ahead of the crack tip, the region of integration reduces to a triangular region and the stress in the plane of the crack can thus be determined without difficulty. However, for material points not on the crack tip line, the region of integration is very complicated and careful analysis is needed. Scattering of plane harmonic waves by a running crack of finite length was investigated by Chen and Sih (1975). They found the dynamic stress intensity factors and crack opening displacements of the finite crack. Exact transient closed form solutions for a stationary semi-infinite crack subjected to a suddenly applied dynamic body force in an unbounded medium have been obtained by Tsai and Ma (1992) for the inplane case and by Ma and Chen (1993) for the anti-plane case. They determined the transient full field solutions by superimposing a fundamental solution in the Laplace transform domain. The fundamental solution used in the problem is an exponentially distributed traction in the Laplace transform domain on the crack faces. This fundamental solution has also successfully been applied to solve the problems of a half plane containing a semi-infinite inclined crack by Tsai and Ma (1993), and Ma and Chen (1994) for inplane and anti-plane problems, respectively.

In this study, a situation in which the configuration of a solid body itself possesses a characteristic length is investigated. We consider the transient response of a stationary finite crack subjected to an incident plane horizontally polarized shear wave as shown in Fig. 1. In analyzing this problem, the waves diffracted by two crack tips will generate an infinite number of waves and make the analysis extremely difficult. It is impossible to solve this complicated problem by direct application of the standard Wiener-Hopf technique, so another approach must be followed. Two useful fundamental problems are proposed to overcome these difficulties. The proposed fundamental problems, which form a key element in the analysis, are solved exactly by the Wiener-Hopf method. The compact and explicit 

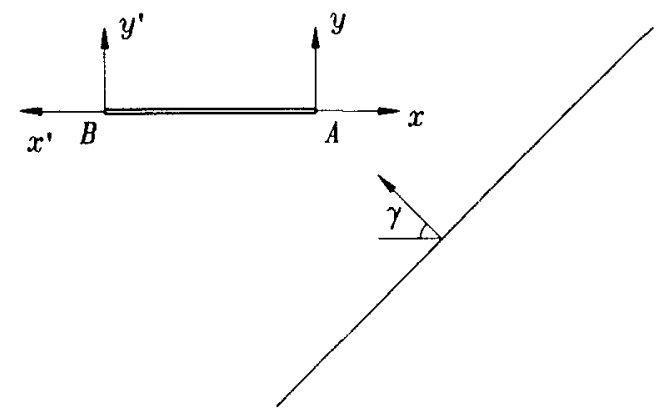

Fig. 1. Configuration and coordinate systems of a finite crack in an unbounded medium.

close form solutions are extracted by means of superposition argument and every term expressed in the solutions has its own physical meaning. Since the stress intensity factor is the key parameter in characterizing dynamic crack growth, we will focus our attention mainly on the determination of the dynamic stress intensity factor. It has been shown from the literature that the dynamic stress intensity factors for plane stress wave diffraction by a crack may be larger than the corresponding static values. This phenomenon, known as dynamic overshoot, is an important subject in the dynamic fracture analysis. In this study, we have found that the dynamic stress intensity factor generated at a finite stationary crack by a plane polarized step pulse with arbitrary incident angle, decreases from its maximum value upon arrival of secondary diffractions and oscillates about the corresponding static values. These results, however, are different from those obtained by Brock (1975) in analyzing the similar problem. The advantages of our treatment over Brock will become clear in the following section of mathematical analysis. These are: (1) the analysis is relatively simple and straightforward, and (2) more importantly, our treatment is easier to obtain the scattering field that is not on the crack tip line.

\section{PROPOSED FUNDAMENTAL PROBLEMS AND FUNDAMENTAL SOLUTIONS}

Two alternative fundamental problems are proposed in this section and the associated fundamental solutions will be used to solve the problem of a finite crack subjected to plane polarized shear waves. The solutions of an exponentially distributed traction applied at the crack faces and exponentially distributed screw dislocations along the crack tip line in the Laplace transform domain will be referred to as the fundamental solutions. The diffracted waves generated from the crack tips can be constructed by superimposing the fundamental solutions in the Laplace transform domain.

The governing equation for the anti-plane problem is represented by the two-dimensional wave equation

$$
\frac{\partial^{2} w}{\partial x^{2}}+\frac{\partial^{2} w}{\partial y^{2}}=b^{2} \frac{\partial^{2} w}{\partial t^{2}}
$$

where $w$ is the out-of-plane displacement and $b$ is the slowness of the shear wave given by

$$
b=\frac{1}{v}=\sqrt{\frac{\rho}{\mu}} .
$$

Here $v$ is the shear wave speed, $\mu$ and $\rho$ are the shear modulus and the mass density of the material, respectively. The nonvanishing shear stresses are 


$$
\tau_{y z}=\mu \frac{\partial w}{\partial y}, \quad \tau_{x z}=\mu \frac{\partial w}{\partial x} .
$$

\subsection{Fundamental solution of distributed loads on crack faces}

Exponentially distributed tractions in the Laplace transform domain for anti-plane deformation are applied on the upper and lower crack faces. Because the tractions are equal but opposite on the two crack faces, the problem can be viewed as a half-plane problem with the material occupying the region $y \geqslant 0$, subjected to the following mixed boundary conditions in the Laplace transform domain

$$
\begin{array}{cc}
\bar{\tau}_{y z}(x, 0, s)=\mathrm{e}^{s \eta x} & \text { for }-\infty<x<0, \\
\bar{w}(x, 0, s)=0 & \text { for } 0<x<\infty .
\end{array}
$$

The Laplace transform parameter $s$ is taken as a positive number and $\eta$ is a constant. The overbar symbol is used for denoting the transform on time $t$. This fundamental problem can be solved by the application of the standard integral transform method. Applying the one-sided Laplace transform over time, the two-sided Laplace transform over $x$ under the restriction of $\operatorname{Re}(\eta)>\operatorname{Re}(\lambda)$, finally the Wiener-Hopf technique is implemented. The solutions of stresses and displacement in the Laplace transform domain, for the boundary conditions (3) and (4), can be expressed as follows

$$
\begin{gathered}
\bar{\tau}_{y z}(x, y, s)=\frac{1}{2 \pi \mathrm{i}} \int_{\Gamma_{\lambda}} \frac{(b+\lambda)^{1 / 2} \mathrm{e}^{-s(x y-\lambda x)}}{(b+\eta)^{1 / 2}(\eta-\lambda)} \mathrm{d} \lambda, \\
\bar{\tau}_{x z}(x, y, s)=\frac{-1}{2 \pi \mathrm{i}} \int_{\Gamma_{j}} \frac{\lambda \mathrm{e}^{-s(x y-\lambda x)}}{(b+\eta)^{1 / 2}(\eta-\lambda)(b-\lambda)^{1 / 2}} \mathrm{~d} \lambda, \\
\bar{w}(x, y, s)=\frac{-1}{2 \pi \mathrm{i}} \int_{\Gamma_{\lambda}} \frac{\mathrm{e}^{-s(x y-\lambda x)}}{\mu s(b+\eta)^{1 / 2}(\eta-\lambda)(b-\lambda)^{1 / 2}} \mathrm{~d} \lambda,
\end{gathered}
$$

where

$$
\alpha(\lambda)=\alpha_{+}(\lambda) \alpha_{-}(\lambda)=(b+\lambda)^{1 / 2}(b-\lambda)^{1 / 2} .
$$

To ensure $\operatorname{Re}(\alpha) \geqslant 0$ everywhere in the $\lambda$-plane, branch cuts are introduced from $b$ to $\infty$, and $-b$ to $-\infty$. The corresponding result of the dynamic stress intensity factor expressed in the Laplace transform domain is

$$
\begin{aligned}
\bar{K}(s) & =\lim _{x \rightarrow 0} \sqrt{2 \pi x} \bar{\tau}_{y z}(x, 0, s) \\
& =-\frac{\sqrt{2}}{\sqrt{s}(b+\eta)^{1 / 2}}
\end{aligned}
$$

2.2. Fundamental solution of screw dislocation distributed along the crack tip line

Consider a semi-infinite crack contained in an unbounded medium. A distributed screw dislocation ahead of the crack tip line yields the following boundary conditions in the Laplace transform domain

$$
\begin{gathered}
\bar{w}(x, 0, s)=\mathrm{e}^{s \eta x} \quad \text { for } 0<x<\infty, \\
\bar{\tau}_{y z}(x, 0, s)=0 \quad \text { for }-\infty<x<0 .
\end{gathered}
$$


The particular problem posed can be solved by means of the Wiener-Hopf method. The solutions of stresses and the displacement expressed in the Laplace transform domain are

$$
\begin{gathered}
\bar{\tau}_{y z}(x, y, s)=\frac{1}{2 \pi \mathrm{i}} \int_{\Gamma_{i}} \frac{\mu s(b-\eta)^{1 / 2}(b+\lambda)^{1 / 2} \mathrm{e}^{-s(x y-\lambda x)}}{(\eta-\lambda)} \mathrm{d} \lambda, \\
\bar{\tau}_{x z}(x, y, s)=\frac{-1}{2 \pi \mathrm{i}} \int_{\Gamma_{i}} \frac{\mu s(b-\eta)^{1 / 2} \mathrm{e}^{-s(x y-\lambda x)}}{(\eta-\lambda)(b-\lambda)^{1 / 2}} \mathrm{~d} \lambda, \\
\bar{w}(x, y, s)=\frac{-1}{2 \pi \mathrm{i}} \int_{\Gamma ;} \frac{(b-\eta)^{1 / 2} \mathrm{e}^{-s(\alpha y-\lambda x)}}{(\eta-\lambda)(b-\lambda)^{1 / 2}} \mathrm{~d} \lambda .
\end{gathered}
$$

The corresponding result of stress intensity factor expressed in the Laplace transform domain is

$$
\bar{K}(s)=-\mu \sqrt{2 s}(b-\eta)^{1 / 2}
$$

\section{DYNAMIC STRESS INTENSITY FACTORS CAUSED BY A HORIZONTALLY POLARIZED SHEAR WAVE}

The idea of an elastic stress intensity factor is a well-established concept in fracture mechanics, and it represents the cornerstone of applied linear elastic fracture mechanics. We will focus our attentions in this study mainly on the evaluation of the dynamic stress intensity factor.

A specific geometry to be consider here is an infinite medium containing a finite crack of length $l$ as shown in Fig. 1. The origins of two coordinate systems $(x, y)$ and $\left(x^{\prime}, y^{\prime}\right)$ are located at crack tips A and B, respectively. The incident plane wave with an incident angle $\gamma$ is represented by the general form

$$
w^{\mathrm{i}}(x, y, t)=F(t+b x \cos \gamma-b y \sin \gamma)
$$

where

$$
F(t)=H(t) \int_{0}^{t} f(\tau) \mathrm{d} \tau
$$

in which $F$ is identically zero when its argument is negative, but is otherwise an arbitrary wave form. Thus, the medium ahead of the incident plane wave front is undisturbed. In eqn (16), $\mathrm{H}()$ denotes the Heaviside step function and $\gamma$ is the angle of the negative $x$-axis and the normal to the wavefront. The position of the wavefront for time $t<0$ is also shown in Fig. 1. Here the angle $\gamma$ is restricted to the range $0<\gamma \leqslant \pi / 2$.

At time $t=0$, the incident plane wavefront strikes the crack tip A and will generate plane reflected and cylindrical diffracted waves. Some time later, i.e. $t=b l \cos \gamma$, the incident plane wave will arrive at the crack tip $B$ and another diffracted wave will be induced. The diffracted waves will scatter back and forth between the crack tips A and B at a later time. In analyzing this problem, the diffractions of stress waves by two crack tips will generate an infinite number of waves, and this must be taken into account.

An effective superposition scheme will be proposed in this study to solve this complicated problem. The incident horizontally polarized shear wave expressed in eqn (15) will give rise to the following shear stress in the infinite medium:

$$
\tau_{y z}^{\mathrm{i}}(x, y, t)=-\mu b \sin \gamma f(t+b x \cos \gamma-b y \sin \gamma) \mathrm{H}(t+b x \cos \gamma-b y \sin \gamma) .
$$

The incident stress field (17) can be represented in the Laplace transform domain as 


$$
\bar{\tau}_{y z}^{\mathrm{i}}(x, y, s)=\frac{1}{2 \pi \mathrm{i}} \int_{\Gamma_{i}} \frac{\mu b \sin \gamma \bar{f}(s)}{\lambda-b \cos \gamma} \mathrm{e}^{-s \lambda y \tan \gamma+s \lambda x} \mathrm{~d} \lambda,
$$

or expressed in the $\left(x^{\prime}, y^{\prime}\right)$ coordinate system as

$$
\bar{\tau}_{y^{\prime} z^{\prime}}^{\mathrm{i}}\left(x^{\prime}, y^{\prime}, s\right)=\frac{1}{2 \pi \mathrm{i}} \int_{\Gamma_{i}^{\prime}} \frac{\mu b \sin \gamma \bar{f}(s)}{\lambda+b \cos \gamma} \mathrm{e}^{\mathrm{s} \lambda y^{\prime} \tan ;+\operatorname{si}\left(x^{\prime}+l\right)} \mathrm{d} \lambda
$$

Having the same definition as in the previous section, the overbar symbol of $f$ is used for denoting the Laplace transform at time $t$. Before the incident stress wave diffracted from the crack tip B, the stress field is precisely the same as that derived for a semi-infinite crack which lies in the plane $y=0$ and $-\infty<x<0$, and is struck by the same incident plane wave. The incident stress field $\bar{\tau}_{y z}^{i}(x, 0, s)$ at $y=0$ generated by the horizontally polarized shear wave is

$$
\bar{\tau}_{y z}^{\mathrm{i}}(x, 0, s)=\frac{1}{2 \pi \mathrm{i}} \int_{\Gamma_{i}} \frac{\mu b \sin \gamma \bar{f}}{\lambda-b \cos \gamma} \mathrm{e}^{s \lambda x} \mathrm{~d} \lambda .
$$

The applied traction on the crack face, in order to eliminate the incident wave as indicated in eqn (20), has the functional form $\mathrm{e}^{s \lambda x}$. Since the solutions of applying traction $\mathrm{e}^{s \eta x}$ on crack faces have been solved in Section 2, the reflected and diffracted fields can be constructed by superimposing the incident wave traction that is equal and opposite to eqn (20). When we combine eqns (7) and (20), the solution of displacement $\bar{w}^{\mathrm{Al}}$ for Al wave (the first wave diffracted from the crack tip A) in the upper plane can be expressed in the Laplace transform domain as follows

$$
\begin{aligned}
\bar{w}^{\mathrm{A} 1}(x, y, s) & =\frac{-1}{2 \pi \mathrm{i}} \int_{\Gamma_{\eta_{1}}} \frac{\mu b \sin \gamma \bar{f}}{\eta_{1}-b \cos \gamma} \frac{1}{2 \pi \mathrm{i}} \int_{\Gamma_{\eta_{2}}} \frac{-\mathrm{e}^{-s x y+s \eta_{2} x}}{\mu s \alpha_{+}\left(\eta_{1}\right)\left(\eta_{1}-\eta_{2}\right) \alpha_{-}\left(\eta_{2}\right)} \mathrm{d} \eta_{2} \mathrm{~d} \eta_{1} \\
& =\frac{1}{2 \pi \mathrm{i}} \int_{\Gamma_{i}} \frac{\sqrt{2 b} \sin (\gamma / 2) \bar{f}^{-s \alpha y+s i x}}{s \alpha_{-}(\lambda)(\lambda-b \cos \gamma)} \mathrm{d} \lambda .
\end{aligned}
$$

The corresponding stress intensity factor expressed in the Laplace transform domain is

$$
\begin{aligned}
\bar{K}^{\mathrm{Al}}(s) & =\frac{-1}{2 \pi \mathrm{i}} \int_{\Gamma_{\dot{\lambda}}} \frac{\mu b \sin \gamma \bar{f}}{\lambda-b \cos \gamma}\left\{\frac{-\sqrt{2}}{\sqrt{s} \alpha_{+}(\lambda)}\right\} \mathrm{d} \lambda \\
& =\frac{-2 \sqrt{b} \mu \sin (\gamma / 2) \vec{f}}{\sqrt{s}} .
\end{aligned}
$$

By using the Cagniard-de Hoop method of Laplace inversion, the dynamic stress intensity factor at the crack tip A induced by the incident $\bar{\tau}_{y z}^{\mathrm{i}}$ wave expressed in time domain will be

$$
K^{\mathrm{Al}}(t)=-2 \sqrt{\frac{b}{\pi}} \mu \sin (\gamma / 2) \int_{0}^{t} \frac{f(\tau)}{\sqrt{t-\tau}} \mathrm{d} \tau
$$

Subsequently, the incident plane wave will propagate toward the crack tip B and will be diffracted at $t=b l \cos \gamma$. Following the similar procedure that is used for constructing the Al wave, the B1 wave (the first wave diffracted from the crack tip B) can be constructed in the coordinate system $\left(x^{\prime}, y^{\prime}\right)$ by eqns (19) and (7) as follows 


$$
\bar{w}^{\mathrm{B} 1}\left(x^{\prime}, y^{\prime}, s\right)=\frac{-1}{2 \pi \mathrm{i}} \int_{\mathrm{r}_{j}} \frac{\sqrt{2 b} \cos (\gamma / 2) \overline{f e}^{-s b l \cos \gamma} \mathrm{e}^{-s x \gamma^{\prime}+s i x^{\prime}}}{s \alpha_{-}(\lambda)(\lambda+b \cos \gamma)} \mathrm{d} \lambda .
$$

The corresponding stress intensity factor at crack B induced by incident plane wave is

$$
\bar{K}^{\mathrm{B} 1}(s)=\frac{2 \sqrt{b} \mu \cos (\gamma / 2) \overline{\mathrm{e}}^{-s h l \cos \gamma}}{\sqrt{s}} .
$$

The dynamic stress intensity factor at the crack tip B expressed in time domain will be

$$
K^{\mathrm{Bl}}(t)=2 \sqrt{\frac{b}{\pi}} \mu \cos (\gamma / 2) \int_{0}^{t-b / \cos ;} \frac{f(\tau)}{\sqrt{t-b l \cos \gamma-\tau}} \mathrm{d} \tau .
$$

As a specific example, if we consider an incident step-stress wave for which

$$
f(t)=\frac{\tau_{0}}{\mu b},
$$

then the integrals in eqns (23) and (26) can easily be evaluated to yield

$$
K_{s}^{\mathrm{A} 1}(t)=-4 \tau_{0} \sqrt{\frac{t}{\pi b}} \sin (\gamma / 2) \mathrm{H}(t)
$$

and

$$
K_{s}^{\mathrm{B} 1}(t)=4 \tau_{0} \sqrt{\frac{t-b l \cos \gamma}{\pi b}} \cos (\gamma / 2) \mathrm{H}(t-b l \cos \gamma)
$$

The results expressed in eqns (28) and (29) are well-known solutions of dynamic stress intensity factor for the first two diffractions of a step-stress wave by a finite crack in an unbounded medium. The analogous solutions have also been found by Achenbach (1970a).

After the first incident wave arrived at crack tip A, the second wave which passes $A$ is the one that is induced by the incident wave and arrives at the left tip $B$, generates the diffracted $\mathrm{B} 1$ wave, and then propagates toward $\mathrm{A}$. When the diffracted $\mathrm{B} 1$ wave arrives at the right tip of the finite crack at time $t=b l(1+\cos \gamma)$, it carries a discontinuous displacement in the $z$-direction which violates the boundary condition for $x>0$. In order to satisfy the boundary condition where the displacement must be continuous for $x>0$, a distributed screw dislocation is required to close the opening displacement. The diffracted A2 wave will be induced when the B1 wave arrived at the crack tip A at time $t=b l(1+\cos \gamma)$. To construct the $\mathrm{A} 2$ wave, we change the formulation for the $\mathrm{B} 1$ wave from $\left(x^{\prime}, y^{\prime}\right)$ to $(x, y)$ coordinate system, then the displacement we must eliminate ahead of the right tip $\mathrm{A}$ is

$$
\bar{w}^{\mathbf{B} 1}(x, 0, s)=\frac{-1}{2 \pi \mathrm{i}} \int_{\Gamma_{i}} \frac{\sqrt{2 b} \cos (\gamma / 2) \bar{f} \mathrm{e}^{-s b l \cos \eta} \mathrm{e}^{s \lambda(x+l)}}{s \alpha_{-}(-\lambda)(\lambda-b \cos \gamma)} \mathrm{d} \lambda .
$$

Again we treat the crack as a semi-infinite crack which lies along the line $y=0$, $-\infty<x<0$. The diffracted A2 wave can be obtained by superimposing the distributed dislocation that is equal and opposite to eqn (30) ahead of the tip $x>0$ in the Laplace transform domain as follows 


$$
\begin{aligned}
\bar{w}^{\mathrm{A} 2}(x, y, s) & =\frac{1}{2 \pi \mathrm{i}} \int_{\Gamma_{\eta_{1}}} \frac{\sqrt{2 b} \cos (\gamma / 2) \bar{f} \overline{\mathrm{e}}^{-s b l \cos \gamma} \mathrm{e}^{s \eta_{1} l}}{s \alpha_{+}\left(\eta_{1}\right)\left(\eta_{1}-b \cos \gamma\right)} \frac{1}{2 \pi \mathrm{i}} \int_{\Gamma_{\eta_{2}}} \frac{-\alpha_{-}\left(\eta_{1}\right) \mathrm{e}^{-s \alpha y+s \lambda x}}{\left(\eta_{1}-\eta_{2}\right) \alpha_{-}\left(\eta_{2}\right)} \mathrm{d} \eta_{2} \mathrm{~d} \eta_{1} \\
& =\frac{-1}{(2 \pi \mathrm{i})^{2}} \int_{\Gamma_{\eta_{1}}} \int_{\Gamma_{\eta_{2}}} \frac{\sqrt{2 b} \cos (\gamma / 2) \bar{f}^{-s b l \cos \gamma} \alpha_{-}\left(\eta_{1}\right) \mathrm{e}^{s \eta_{1} l} \mathrm{e}^{-s \alpha y+s \eta_{2} x}}{s \alpha_{+}\left(\eta_{1}\right)\left(\eta_{1}-b \cos \gamma\right)\left(\eta_{1}-\eta_{2}\right) \alpha_{-}\left(\eta_{2}\right)} \mathrm{d} \eta_{2} \mathrm{~d} \eta_{1} .
\end{aligned}
$$

Here eqn (13) has been used to construct the solution of the diffracted A2 wave.

Using eqns (30) and (14), the corresponding stress intensity factor at crack tip A expressed in the Laplace transform domain will be

$$
\bar{K}^{\mathrm{A} 2}(s)=\frac{-1}{2 \pi \mathrm{i}} \int_{\Gamma_{j}} \frac{2 \sqrt{b} \mu \cos (\gamma / 2) \bar{f} \mathrm{e}^{-s b l \cos \gamma} \alpha_{-}(\lambda) \mathrm{e}^{\mathrm{s} \lambda /}}{\sqrt{s} \alpha_{+}(\lambda)(\lambda-b \cos \gamma)} \mathrm{d} \lambda .
$$

Inversion of the Laplace transform yields

$$
K^{\mathrm{A} 2}(t)=\frac{2 \sqrt{b} \mu \cos (\gamma / 2)}{\pi^{3 / 2}} \int_{b l}^{t-b / \cos \gamma} \int_{0}^{t-\tau-b l \cos \gamma} \frac{f(\delta) \sqrt{\tau+b l}}{\sqrt{t-\tau-b l \cos \gamma-\delta} \sqrt{\tau-b l}(\tau+b l \cos \gamma)} \mathrm{d} \delta \mathrm{d} \tau .
$$

Similarly, we use the same process that is used for constructing the A2 wave, the diffracted B2 wave, which occurs after the diffracted A1 wave arrives at crack tip B at $t=b l$, can be obtained from eqns (21) and (13), and is expressed in the coordinate system of $\left(x^{\prime}, y^{\prime}\right)$ as follows

$$
\begin{aligned}
& \bar{w}^{\mathrm{B} 2}\left(x^{\prime}, y^{\prime}, s\right)=\frac{-1}{2 \pi \mathrm{i}} \int_{\Gamma_{\eta_{1}}} \frac{\sqrt{2 b} \sin (\gamma / 2) \overline{\mathrm{e}}^{s \eta_{1} !}}{s \alpha_{+}\left(\eta_{1}\right)\left(\eta_{1}+b \cos \gamma\right)} \frac{1}{2 \pi \mathrm{i}} \int_{\Gamma_{\eta_{:}}} \frac{-\alpha_{-}\left(\eta_{1}\right) \mathrm{e}^{-s \alpha \gamma^{\prime}+s \lambda x^{\prime}}}{\left(\eta_{1}-\eta_{2}\right) \alpha_{-}\left(\eta_{2}\right)} \mathrm{d} \eta_{2} \mathrm{~d} \eta_{1} \\
& =\frac{1}{(2 \pi \mathrm{i})^{2}} \int_{\Gamma_{\eta_{1}}} \int_{\Gamma_{\eta_{2}}} \frac{\sqrt{2 b} \sin (\gamma / 2) \bar{f} \alpha_{-}\left(\eta_{1}\right) \mathrm{e}^{s \eta_{1} /} \mathrm{e}^{-s \alpha y^{\prime}+s \eta_{2} x^{\prime}}}{\left.s \eta_{1}\right)\left(\eta_{1}+b \cos \gamma\right)\left(\eta_{1}-\eta_{2}\right) \alpha_{-}\left(\eta_{2}\right)} \mathrm{d} \eta_{2} \mathrm{~d} \eta_{1} .
\end{aligned}
$$

The correspondent stress intensity factor induced by the A1 wave at the crack tip B can be expressed in the Laplace transform domain as follows

$$
\bar{K}^{\mathrm{B} 2}(s)=\frac{1}{2 \pi \mathrm{i}} \int_{\Gamma_{j},} \frac{2 \sqrt{b} \mu \sin (\gamma / 2) \bar{f} \alpha_{-}(\hat{\lambda}) \mathrm{e}^{s i \lambda}}{\sqrt{s} \alpha_{+}(\lambda)(\lambda+b \cos \gamma)} \mathrm{d} \lambda
$$

The inverse Laplace transform of eqn (35) is

$$
K^{\mathrm{B} 2}(t)=\frac{-2 \sqrt{b} \mu \sin (\gamma / 2)}{\pi^{3 / 2}} \int_{b l}^{t} \int_{0}^{t-\tau} \frac{f(\delta) \sqrt{\tau+b l}}{\sqrt{t-\tau-\delta} \sqrt{\tau-b l}(\tau-b l \cos \gamma)} \mathrm{d} \delta \mathrm{d} \tau
$$

Consider a step-stress incident wave, eqns (38) and (36) will become

$$
K_{s}^{\mathrm{A} 2}(t)=\frac{4 \tau_{0} \cos (\gamma / 2)}{\sqrt{b} \pi^{3 / 2}} \int_{b l}^{t-b l \cos \gamma} \frac{\sqrt{t-\tau-b l \cos \gamma} \sqrt{\tau+b l}}{\sqrt{\tau-b l}(\tau+b l \cos \gamma)} \mathrm{d} \tau
$$

and 


$$
K_{s}^{\mathrm{B} 2}(t)=\frac{-4 \tau_{0} \sin (\gamma / 2)}{\sqrt{b} \pi^{3 / 2}} \int_{b l}^{t} \frac{\sqrt{t-\tau} \sqrt{\tau+b l}}{\sqrt{\tau-b l}(\tau-b l \cos \gamma)} \mathrm{d} \tau .
$$

The B2 wave, which is generated by diffracting the A1 wave at the left tip of the crack, will return to tip A at $t=2 b l$. After it arrives at tip A, the displacement boundary condition for $x>0$ will be violated again. As we did before, an appropriate sequence of screw dislocations along $x>0$ must be superimposed to close the opening displacement ahead of the crack tip. Combine the results of eqns (14) and (34), the corresponding stress intensity factor for tip A in the Laplace transform domain can be obtained as follows

$$
\bar{K}^{\mathrm{A} 3}(s)=\frac{-1}{(2 \pi \mathrm{i})^{2}} \int_{\Gamma_{\eta_{1}}} \int_{\Gamma_{\eta_{2}}} \frac{2 \sqrt{b} \mu \sin (\gamma / 2) \bar{f} \alpha_{-}\left(\eta_{1}\right) G\left(\eta_{1}, \eta_{2}\right) \mathrm{e}^{s \eta_{1} l} \mathrm{e}^{s \eta_{2} l}}{\sqrt{s \alpha_{+}\left(\eta_{1}\right)\left(\eta_{1}+b \cos \gamma\right)}} \mathrm{d} \eta_{2} \mathrm{~d} \eta_{1},
$$

where

$$
G\left(\eta_{1}, \eta_{2}\right)=\frac{\alpha_{-}\left(\eta_{2}\right)}{\left(\eta_{1}+\eta_{2}\right) \alpha_{-}\left(-\eta_{2}\right)}
$$

Applying the inverse Laplace transform to eqn (39), the stress intensity factor in the time domain is

$$
\begin{aligned}
K^{\mathrm{A} 3}(t)= & \frac{-2 \sqrt{b} \mu \sin (\gamma / 2)}{\pi^{5 / 2}} \int_{2 b l}^{t} \int_{b l}^{\tau-b l} \int_{0}^{t-\tau} \frac{f(\delta)}{\sqrt{t-\tau-\delta}} \\
& \times\left[\frac{\sqrt{t_{1}+b l} \sqrt{t_{2}+b l}}{\sqrt{t_{1}-b l}\left(t_{1}-b l \cos \gamma\right)\left(t_{1}+t_{2}\right) \sqrt{t_{2}-b l}}\right]_{t=\tau} \mathrm{d} \delta \mathrm{d} t_{1} \mathrm{~d} \tau
\end{aligned}
$$

where

$$
t_{1}+t_{2}=t
$$

Similarly, the stress intensity factor at the crack tip B due to the influence of the A2 wave at $t=b l(2+\cos \gamma)$ is

$$
\begin{aligned}
K^{\mathrm{B} 3}(t)=\frac{2 \sqrt{b} \mu \cos (\gamma / 2)}{\pi^{5 / 2}} \int_{2 b l}^{t-b l \cos \gamma} & \int_{b l}^{\tau-b l} \int_{0}^{t-\tau-b l \cos \gamma} \frac{f(\delta)}{\sqrt{t-\tau-b l \cos \gamma-\delta}} \\
\times & {\left[\frac{\sqrt{t_{1}+b l} \sqrt{t_{2}+b l}}{\sqrt{t_{1}-b l}\left(t_{1}+b l \cos \gamma\right)\left(t_{1}+t_{2}\right) \sqrt{t_{2}-b l}}\right]_{t=\tau} \mathrm{d} \delta \mathrm{d} t_{1} \mathrm{~d} \tau . }
\end{aligned}
$$

For the step-stress incident wave case, eqns (40) and (41) yield

$$
\begin{aligned}
K_{s}^{\mathrm{A} 3}(t)=\frac{-4 \tau_{0} \sin (\gamma / 2)}{\sqrt{b} \pi^{5 / 2}} \int_{2 b l}^{t} \int_{b l}^{\tau-b l} & \sqrt{t-\tau} \\
\times & {\left[\frac{\sqrt{t_{1}+b l} \sqrt{t_{2}+b l}}{\sqrt{t_{1}-b l}\left(t_{1}-b l \cos \gamma\right)\left(t_{1}+t_{2}\right) \sqrt{t_{2}-b l}}\right]_{t=\tau} \mathrm{d} t_{1} \mathrm{~d} \tau }
\end{aligned}
$$

and

$$
\begin{aligned}
K_{s}^{\mathrm{B} 3}(t)=\frac{4 \tau_{0} \cos (\gamma / 2)}{\sqrt{b} \pi^{5 / 2}} \int_{2 b l}^{t-b l \cos \gamma} \int_{b l}^{t-b l} & \sqrt{t-\tau-b l \cos \gamma} \\
\times & {\left[\frac{\sqrt{t_{1}+b l} \sqrt{t_{2}+b l}}{\sqrt{t_{1}-b l}\left(t_{1}-b l \cos \gamma\right)\left(t_{1}+t_{2}\right) \sqrt{t_{2}-b l}}\right]_{t=\tau} \mathrm{d} t_{1} \mathrm{~d} \tau . }
\end{aligned}
$$


For the time being, we have constructed in detail the first three waves which have contributions to the stress intensity factor at crack tips A and B. The above mentioned process can be continued indefinitely. Following a similar procedure, the complete solutions for dynamic stress intensity factor at tips A and B that account for the contributions of incident waves and all the diffracted waves induced from the two crack tips are finally obtained explicitly. Our results at each crack tip are exact up to an infinite length of time. The complete solutions can be simplified into very compact formulations as follows

$$
\begin{aligned}
& K_{\mathrm{A}}(t)=\sum_{n=1}^{\infty} K^{\mathrm{A} n}(t), \\
& K_{\mathrm{B}}(t)=\sum_{n=1}^{\infty} K^{\mathrm{B} n}(t),
\end{aligned}
$$

where

$$
\begin{aligned}
& K^{\mathrm{A} 1}(t)=-2 \sqrt{\frac{b}{\pi}} \mu \sin (\gamma / 2) \int_{0}^{t} \frac{f(\tau)}{\sqrt{t-\tau}} \mathrm{d} \tau \\
& K^{\mathrm{B} 1}(t)=2 \sqrt{\frac{b}{\pi}} \mu \cos (\gamma / 2) \int_{0}^{t-b l \cos \gamma} \frac{f(\tau)}{\sqrt{t-b l \cos \gamma-\tau}} \mathrm{d} \tau, \\
& K^{\mathrm{A} 2}(t)=\frac{2 \sqrt{b} \mu \cos (\gamma / 2)}{\pi^{3 / 2}} \int_{b l}^{t-b l \cos \gamma} \int_{0}^{t-\tau-b / \cos \gamma} \frac{f(\delta) \sqrt{\tau+b l}}{\sqrt{t-\tau-b l \cos \gamma-\delta} \sqrt{\tau-b l}(\tau+b l \cos \gamma)} \mathrm{d} \delta \mathrm{d} \tau, \\
& K^{\mathrm{B} 2}(t)=\frac{-2 \sqrt{b} \mu \sin (\gamma / 2)}{\pi^{3 / 2}} \int_{b l}^{t} \int_{0}^{t-\tau} \frac{f(\delta) \sqrt{\tau+b l}}{\sqrt{t-\tau-\delta} \sqrt{\tau-b l}(\tau-b l \cos \gamma)} \mathrm{d} \delta \mathrm{d} \tau, \\
& K^{\mathrm{A} n}(t)=\frac{(-1)^{n} 2 \sqrt{b} \mu}{\sqrt{\pi} \pi^{n-1}} \\
& \times \int_{(n-1) b l}^{t-q b l \cos \gamma} \int_{b l}^{a_{1}} \int_{b l}^{a_{2}} \cdots \int_{b l}^{a_{n-2}} \int_{0}^{t-\tau-q b \cos \gamma} \frac{[(1-q) \sin (\gamma / 2)+q \cos (\gamma / 2)] f(\delta)}{\sqrt{t-\tau-q b l \cos \gamma-\delta}} \operatorname{ASIF} \\
& \mathrm{d} \delta \mathrm{d} t_{n-2} \mathrm{~d} t_{n-3} \cdots \mathrm{d} t_{1} \mathrm{~d} \tau, \quad \text { for } n=3,4,5, \cdots \text {, } \\
& K^{\mathrm{B} n}(t)=\frac{(-1)^{n-1} 2 \sqrt{b} \mu}{\sqrt{\pi} \pi^{n-1}} \\
& \times \int_{(n-1) b l}^{t-(1-q) b l \cos \gamma} \int_{b l}^{a_{1}} \int_{b l}^{a_{2}} \cdots \int_{b l}^{a_{n-2}} \int_{0}^{t-\tau-(1-q) b l \cos \gamma} \frac{[(1-q) \cos (\gamma / 2)+q \sin (\gamma / 2)] f(\delta)}{\sqrt{t-\tau-(1-q) b l \cos \gamma-\delta}} \operatorname{BSIF} \\
& \mathrm{d} \delta \mathrm{d} t_{n-2} \mathrm{~d} t_{n-3} \cdots \mathrm{d} t_{1} \mathrm{~d} \tau, \quad \text { for } n=3,4,5, \cdots \text {, }
\end{aligned}
$$

and

$$
\begin{aligned}
& a_{1}=\tau-(n-2) b l, \\
& a_{r}=\tau-t_{1}-t_{2}-\cdots-t_{r-1}-(n-v-1) b l, \quad \text { for } v=2,3,4, \cdots n-2, \\
& t_{1}+t_{2}+t_{3}+\cdots+t_{n}=t, \\
& q=0, \quad \text { when } n=3,5,7, \ldots ; \quad q=1, \quad \text { when } n=4,6,8, \ldots,
\end{aligned}
$$

$\mathrm{ASIF}=$

$$
\left[\frac{\sqrt{t_{1}+b l} \sqrt{t_{2}+b l} \cdots \sqrt{t_{n-1}+b l}}{\sqrt{t_{1}-b l}\left[t_{1}+(-1)^{n} b l \cos \gamma\right]\left(t_{1}+t_{2}\right)\left(t_{2}+t_{3}\right) \cdots\left(t_{n-2}-t_{n-1}\right) \sqrt{t_{2}-b l} \sqrt{t_{3}-b l} \cdots \sqrt{t_{n-1}-b l}}\right]_{t=\tau},
$$


$\mathrm{BSIF}=$

$$
\left[\frac{\sqrt{t_{1}+b l} \sqrt{t_{2}+b l} \cdots \sqrt{t_{n-1}+b l}}{\sqrt{t_{1}-b l}\left[t_{1}+(-1)^{n-1} b l \cos \gamma\right]\left(t_{1}+t_{2}\right)\left(t_{2}+t_{3}\right) \cdots\left(t_{n-2}+t_{n-1}\right) \sqrt{t_{2}-b l} \sqrt{t_{3}-b l} \cdots \sqrt{t_{n-1}-b l}}\right]_{t=\tau} .
$$

If the incident wave is a step-stress wave [i.e. $\left.f=\tau_{0} /(\mu b)\right]$, the solutions can be simplified and yield

$$
\begin{aligned}
& K_{s, \mathrm{~A}}(t)=\sum_{n=1}^{\infty} K_{s}^{\mathrm{A} n}(t), \\
& K_{s, \mathrm{~B}}(t)=\sum_{n=1}^{\infty} K_{s}^{\mathrm{B} n}(t),
\end{aligned}
$$

where

$$
\begin{aligned}
& K_{s}^{\mathrm{Al}}(t)=-4 \tau_{0} \sqrt{\frac{t}{\pi b}} \sin (\gamma / 2) \mathrm{H}(t) \\
& K_{s}^{\mathrm{B} 1}(t)=4 \tau_{0} \sqrt{\frac{t-b l \cos \gamma}{\pi b}} \cos (\gamma / 2) \mathrm{H}(t-b l \cos \gamma), \\
& K_{s}^{\mathrm{A} 2}(t)=\frac{4 \tau_{0} \cos (\gamma / 2)}{\sqrt{b} \pi^{3 / 2}} \int_{b l}^{t-b l \cos \gamma} \frac{\sqrt{t-\tau-b l \cos \gamma} \sqrt{\tau+b l}}{\sqrt{\tau-b l}(\tau+b l \cos \gamma)} \mathrm{d} \tau, \\
& K_{s}^{\mathbf{B} 2}(t)=\frac{-4 \tau_{0} \sin (\gamma / 2)}{\sqrt{b} \pi^{3 / 2}} \int_{b l}^{t} \frac{\sqrt{t-\tau} \sqrt{\tau+b l}}{\sqrt{\tau-b l}(\tau-b l \cos \gamma)} \mathrm{d} \tau, \\
& K_{s}^{\mathrm{An}}(t)=\frac{(-1)^{n} 4 \tau_{0}}{\sqrt{\pi b} \pi^{n-1}} \int_{(n-1) b l}^{t-q b / \cos \gamma} \int_{b l}^{a_{1}} \int_{b l}^{a_{2}} \cdots \int_{b l}^{a_{n-2}}[(1-q) \sin (\gamma / 2) \\
& +q \cos (\gamma / 2)] \sqrt{t-\tau-q b l \cos \gamma} \operatorname{ASIF} \quad \mathrm{d} t_{n-2} \mathrm{~d} t_{n-3} \cdots \mathrm{d} t_{1} \mathrm{~d} \tau, \quad \text { for } n=3,4,5, \cdots, \\
& K_{s}^{\mathrm{B} n}(t)=\frac{(-1)^{n-1} 4 \tau_{0}}{\sqrt{\pi b} \pi^{n-1}} \int_{(n-1) b l}^{t-(1-q) b l \cos \gamma} \int_{h l}^{a_{1}} \int_{b l}^{a_{2}} \cdots \int_{h l}^{a_{n-2}}[(1-q) \cos (\gamma / 2) \\
& +q \sin (\gamma / 2)] \sqrt{t-\tau-(1-q) b l \cos \gamma} \text { BSIF } \quad \mathrm{d} t_{n-2} \mathrm{~d} t_{n-3} \cdots \mathrm{d} t_{1} \mathrm{~d} \tau \text {, for } n=3,4,5, \cdots \text {. }
\end{aligned}
$$

The corresponding static limits of the stress intensity factor for a step-stress wave are

$$
K_{s, \mathrm{~A}}^{s}=-\tau_{0} \sin \gamma \sqrt{\frac{\pi l}{2}}
$$

and

$$
K_{s . \mathrm{B}}^{s}=\tau_{0} \sin \gamma \sqrt{\frac{\pi l}{2}}
$$

In the previous section, the exact transient solutions of dynamic stress intensity factors for diffraction of a horizontally polarized shear wave by a finite crack have been derived. The induced wave fronts of incident and diffracted waves in a short time period are shown 


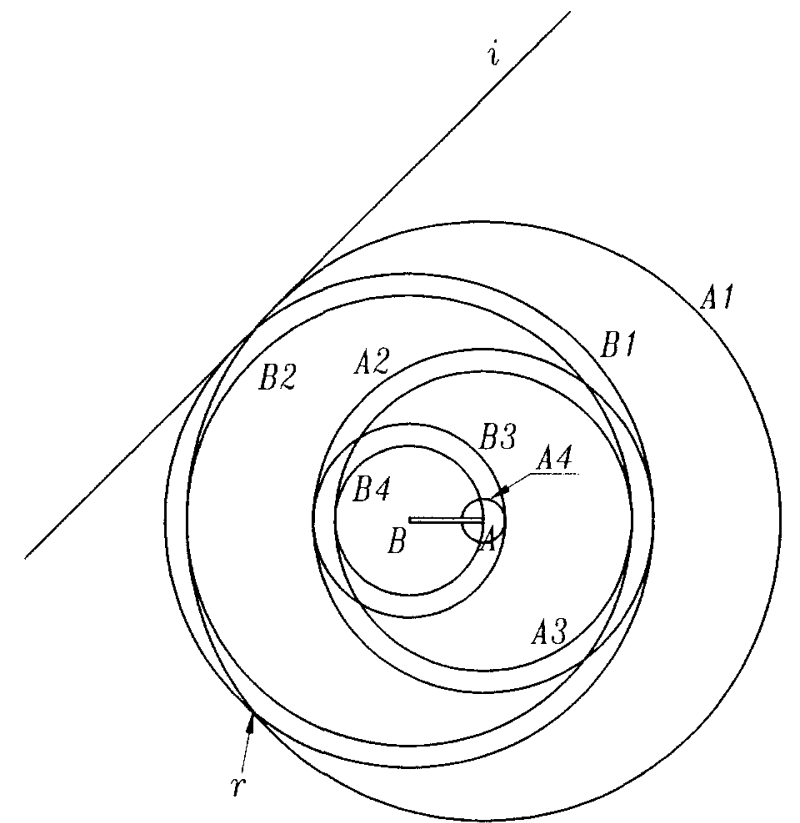

Fig. 2. Wave fronts of the incident and diffracted waves for a short time period.

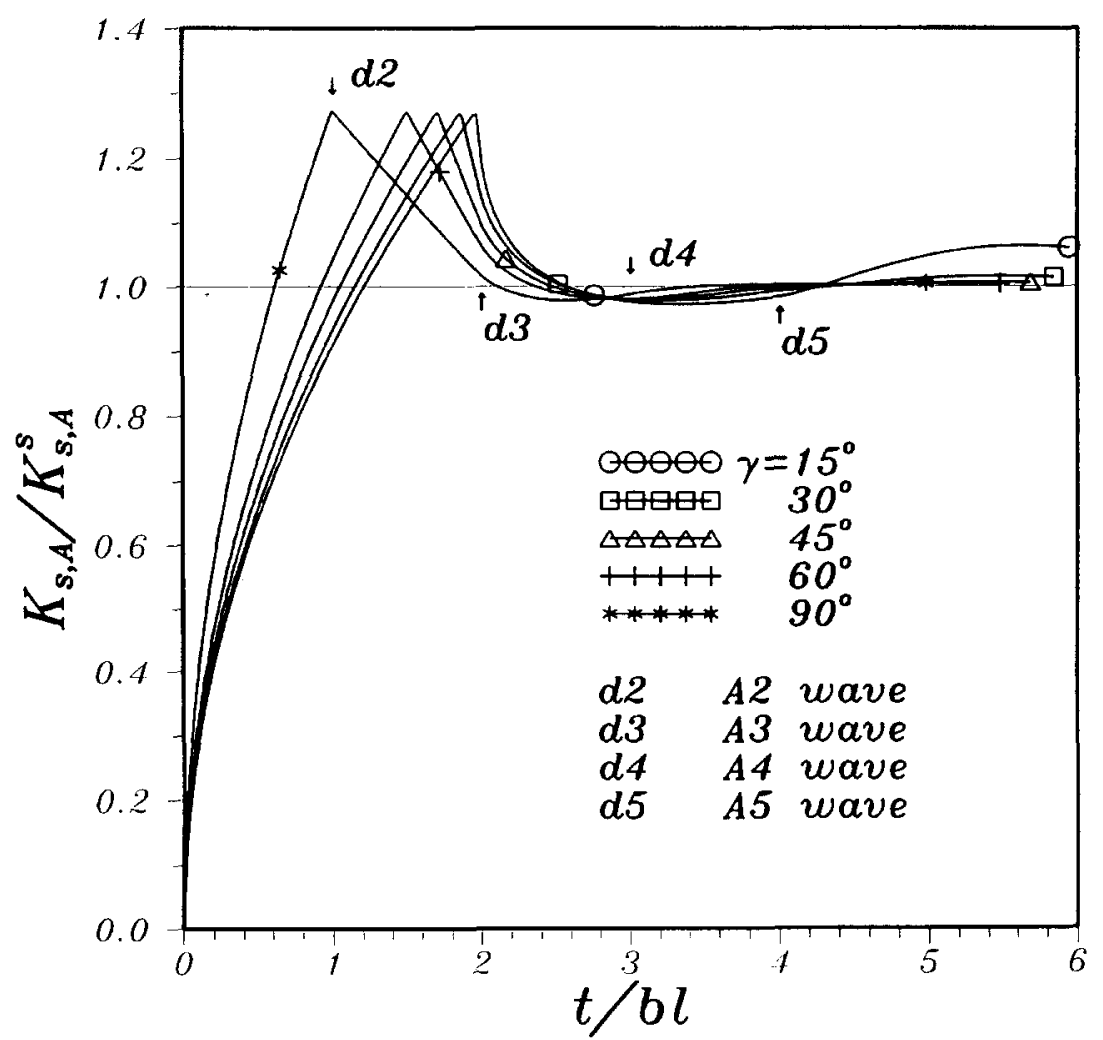

Fig. 3. Transient response of the stress intensity factor normalized with the corresponding static value at tip $\mathrm{A}$ for different values of $\gamma$.

in Fig. 2. Numerical calculations have been done here for a step-stress wave case at both tips of the finite crack. Figures 3 and 4 show the dimensionless stress intensity factors $K_{s, \mathrm{~A}} /$ $K_{s, \mathrm{~A}}^{s}$ and $K_{s, \mathrm{~B}} / K_{s, \mathrm{~B}}^{s}$ vs the dimensionless time $t / b l$ for different values of the incident angle $\gamma$ at tips $\mathrm{A}$ and $\mathrm{B}$, respectively. The corresponding time of the diffracted waves arrival at the tip is also indicated in the figures for the case $\gamma=90^{\circ}$. It indicates in these figures that the dynamic stress factors will increase with time and will reach a maximum overshot at the 


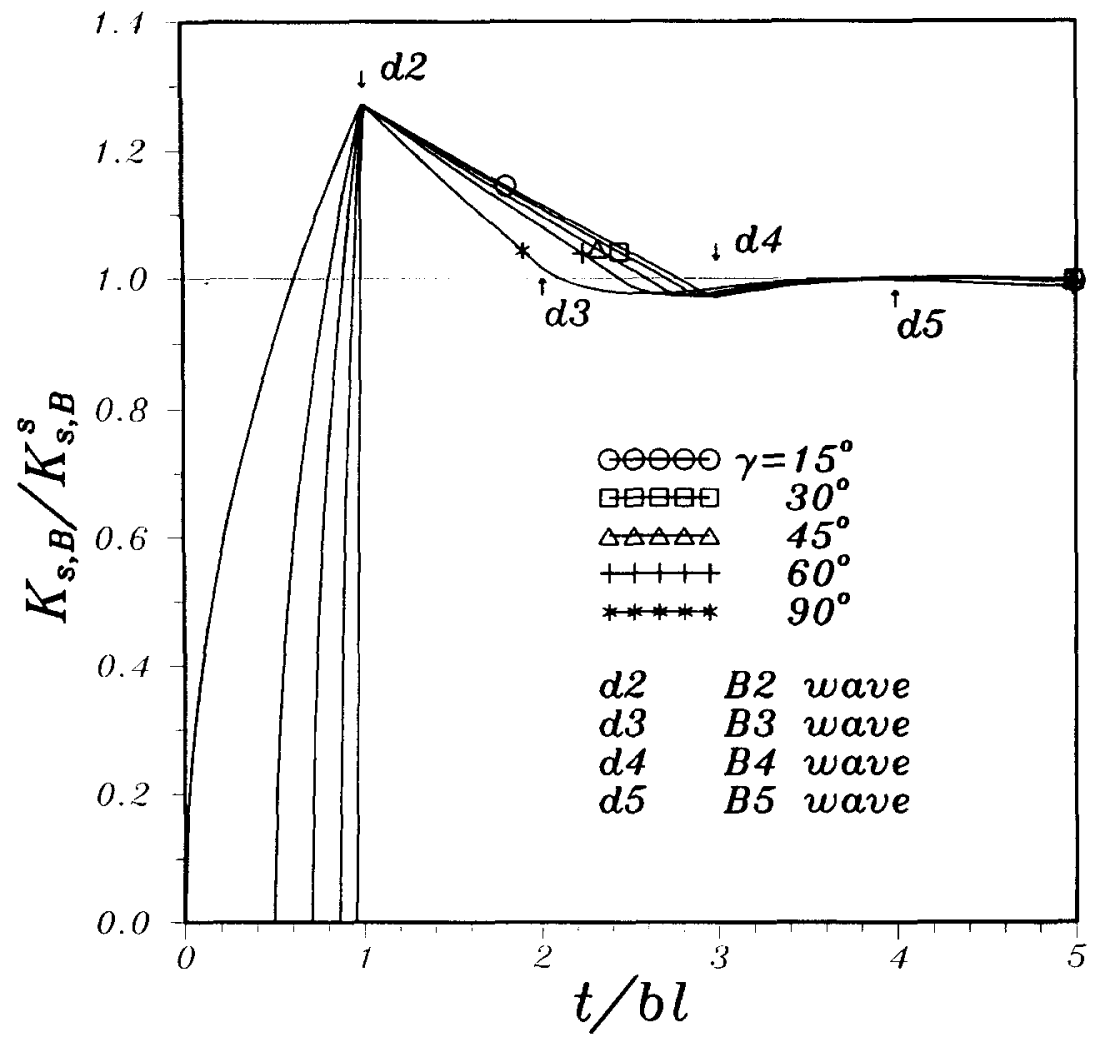

Fig. 4. Transient response of the stress intensity factor normalized with the corresponding static value at tip $B$ for different values of $\gamma$.

instance that the second wave arrives at the crack tip, which is greater than the correspondent static value, and then oscillate near the static value after the first three waves have passed through the crack tip. Moreover, from eqns (28), (29), (48) and (49) we can calculate that the ratio of the value for maximum dynamic overshot and the corresponding static value will always be $4 / \pi$. This is a very important characteristic feature that must be taken into account for the dynamic fracture analysis. Figures 5 and 6 show the dimensionless stress intensity factors $K_{s, \mathrm{~A}} l^{1 / 2} / \tau_{0}$ and $K_{s, \mathrm{~B}} l^{1 / 2} / \tau_{0}$ vs the dimensionless time $t / b l$ for different values of the incident angle $\gamma$ at tips $A$ and $B$, respectively. It can be seen that as the angle $\gamma$ increases, the peak magnitude increases and it will approach a larger static value. It is worthy to note that the stress intensity factors at crack tip B for a small incident angle increase very rapidly after the first wave arrives at the tip. The times that the maximum values of the overshot occur are $b l(1+\cos \gamma)$ and $b l$ for crack tips $\mathrm{A}$ and $\mathrm{B}$, respectively. Substituting $t=b l(1+\cos \gamma)$ and $t=b l$ into eqns (28) and (29), respectively, we find that the maximum value of stress intensity factors for tips $\mathrm{A}$ and $\mathrm{B}$ are equal and $\left|K_{s, \mathrm{~A}}^{\max }\right|=$ $\left|K_{s, \mathrm{~B}}^{\max }\right|=2 \tau_{0}(2 l / \pi)^{1 / 2} \sin \gamma$. Because the time that the stress intensity factor of crack tip B reaches its maximum value is shorter than that of tip $A$, the finite crack will propagate first from tip B for large dynamic fracture toughness. Only for the special case of normal incident $\left(\gamma=90^{\circ}\right)$ will the two crack tips propagate at the same time.

Brock (1975) also investigated the same problem for the long time behavior. His results indicated that the peak dynamic stress intensity factors could occur after the arrival of the second wave ( $d_{2}$ wave), which means that secondary diffractions may produce even higher peaks than the earlier peaking. Numerical results in fig. 8 of Brock (1975) show that higher peaks may be caused by secondary diffraction for small incident angles $\left(\gamma<45^{\circ}\right)$. However, our present results indicate that the maximum dynamic stress intensity factor in the transient period always occurs at the instance that the second wave arrives at crack tip A or B. Hence, fracture of a crack occurs before the arrival or secondary diffractions and the fracture initiation may be examined by short time analysis of a semi-infinite crack. 


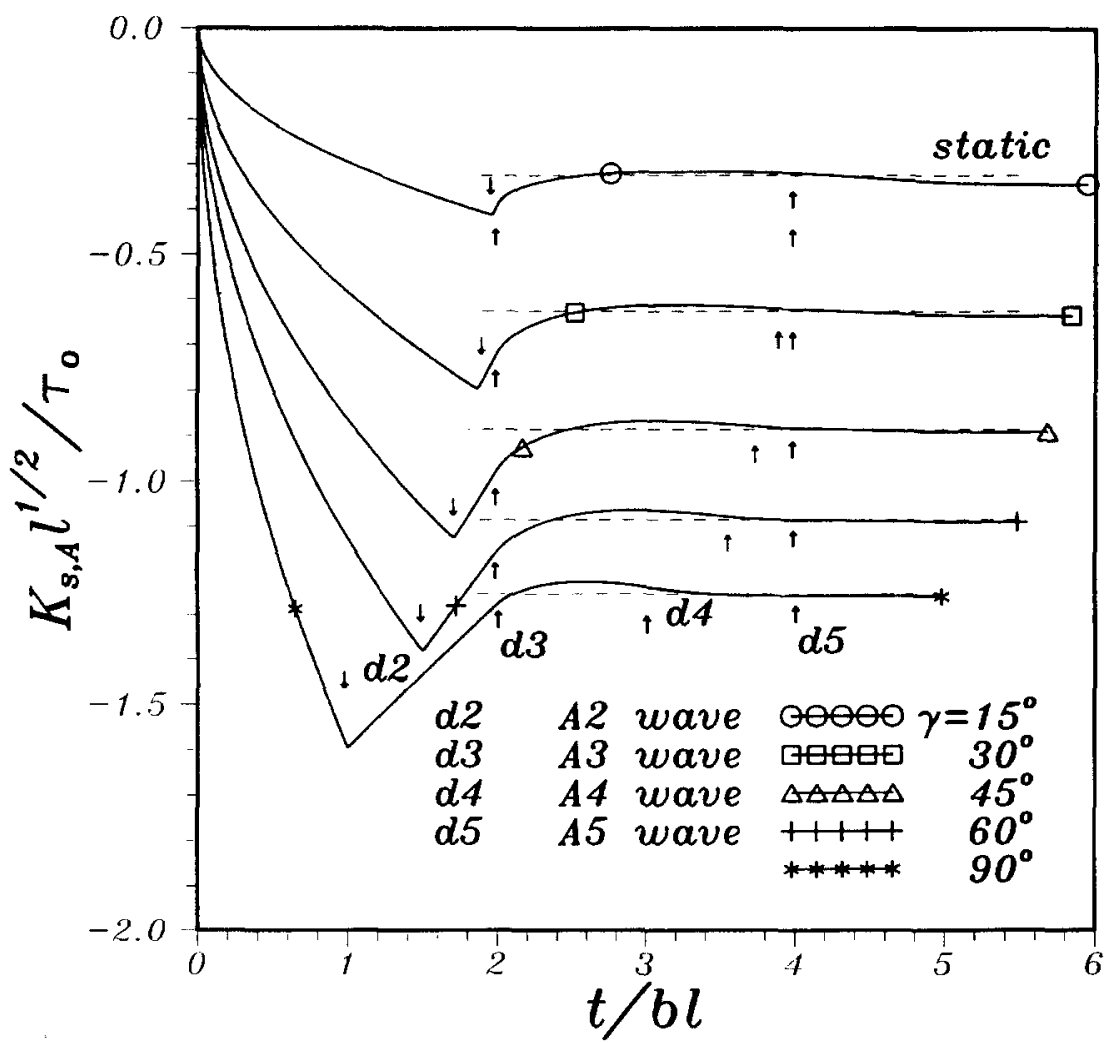

Fig. 5. Transient response of the stress intensity factor at tip A for different values of $\gamma$.

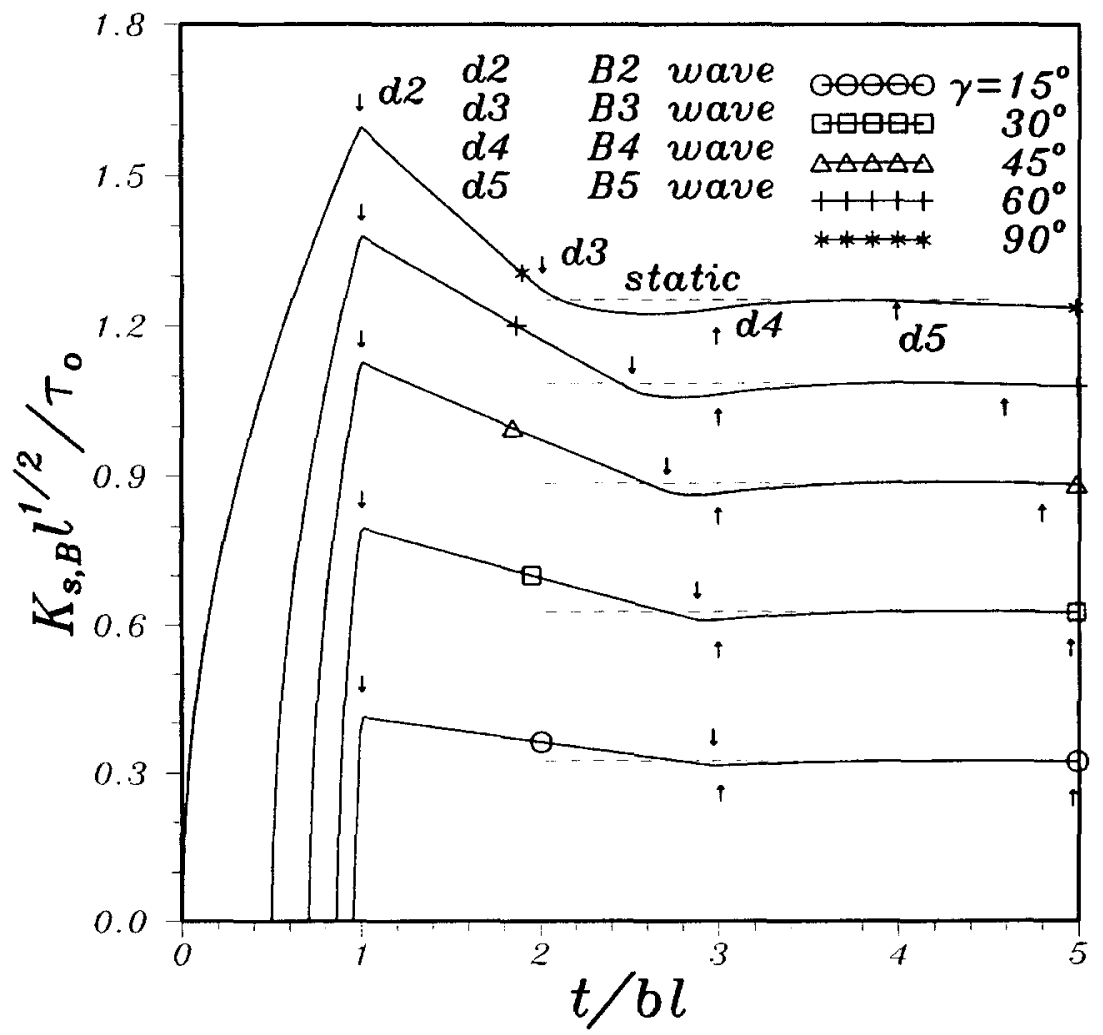

Fig. 6. Transient response of the stress intensity factor at tip B for different values of $\gamma$. 


\section{CONCLUSIONS}

Most of the problems that have been studied in the development of fracture mechanics are quasi-static. Numerous problems have existed for which the assumption that the deformation is quasi-static is invalid and the inertia of the material must be taken into account. Because of the difficulties in mathematical complexity, analytical solutions for an elastic solid containing a finite crack subjected to dynamic loading are very rare. In conventional studies of a semi-infinite crack in an unbounded medium subjected to dynamic loading, the complete solution can be obtained by applying direct integral transform methods. If a dynamic cracked problem having a characteristic length or the loading condition is unsymmetric, then the same procedure cannot be applied directly. In this investigation, we propose a powerful superposition methodology, which is performed in the Laplace transform domain, and successfully applied to solve the transient response of a finite crack contained in an unbounded medium. The finite crack is struck by a horizontally polarized shear wave of arbitrary shape. Two useful fundamental solutions are proposed to solve this problem. The complete closed form solutions for transient stress intensity factor of both crack tips are obtained and expressed in very simple and compact formulations. Every term expressed in the solution has its own physical meaning. The solutions are valid for an infinite length of time and have accounted for the contributions coming from all diffracted waves which propagate back and forth between the two tips.

For the numerical calculation of the transient stress intensity factor, a step-stress incident wave is investigated in detail. It is shown in the numerical results that the dynamic stress intensity factors increase with time at first and will reach a maximum dynamic overshot at the instance that the second wave arrives at the tip, and then oscillate near the static value after the first three waves have passed through the crack tip. It is also concluded in this investigation that the transient effect for the stress intensity factor can be neglected after the first three diffracted waves have passed the crack tip. The ratio of the value for the maximum dynamic overshot and the corresponding static value is always $4 / \pi$ for both tips and for various incident angles. It is also pointed out that fracture tends to occur as the incident angle becomes larger and will onset possibly first at the left crack tip B. The results obtained in this investigation provide much information that is very important for the study on dynamic fracture. The powerful technique used in this paper can be provided for further investigation in more complicated dynamic fracture problems especially on the crack propagation event. Moreover, it is easy to extend the method proposed here to solve more difficult problems which involve interaction of a finite crack with boundaries. A further study related to dynamic fracture based on this method will be given in a follow-up report.

Acknowledgements-The authors gratefully acknowledge the financial support of this research by the National Science Council (Republic of China) under Grant NSC 83-0401-E-002-115.

\section{REFERENCES}

Achenbach, J. D. (1970a) Brittle and ductile extension of a finite crack by a horizontally polarized shear wave. Int. J. Engng Science 8, 947-966.

Achenbach, J. D. (1970b) Extension of a crack by a shear wave. Z. Angew. Math. Phys. 21, 887-900.

Brock, L. M. (1975) Effects of secondary diffractions on the stress intensity factors generated for a finite crack by a shear wave. Int. J. Engng Science 13, 851-859.

Brock, L. M. (1982) Shear and normal impact loading on one face of a narrow slit. Int. J. Solids Structures $\mathbf{1 8}$, 467-477.

Brock, L. M. (1984) Stresses in a surface obstacle undercut due to rapid indentation. J. Elasticity 14, 415-424.

Brock, L. M., Jolles, M. and Schroedl, M. (1985) Dynamic impact over a subsurface crack: applications to the dynamic tear test. J. Appl. Mech. 52, 287-290.

Chen, E. P. and Sih, G. C. (1975) Scattering of plane waves by a propagating crack. J. Appl. Mech. 42, 705-711

Chen, E. P. (1977) Impact response of a finite crack in a finite strip under anti-plane shear. Engng Fracture Mech. 9, 719-724.

Chen, E. P. (1978) Sudden appearance of a crack in a stretched finite strip. J. Appl. Mech. 45, 277-280.

de Hoop, A. T. (1958) Representation theorems for the displacement in an elastic solid and their application to elastodynamic diffraction theory. Doctoral dissertation, Technische hoegschool, Delft.

Flitman, L. M. (1963) Waves generated by sudden crack in a continuous elastic medium. Applied Mathematics and Mechanics (PMM) 27, 938-953. 
Freund, L. B. (1974) The stress intensity factor due to normal impact loading of the faces of a crack. Int. J. Engng Science 12, 179-189.

Freund, L. B. (1990) Dynamic Fracture Mechanics. Cambridge University Press, Cambridge.

Itou, S. (1980) Transient response of a finite crack in a strip with stress-free edges. J. Appl. Mech. 47, 801-805.

Itou, S. (1981) Transient response of a finite crack in a half plane under impact load. J. Appl. Mech. 48, 534-538.

Kostrov, B. V. (1964) Self-similar problems of propagation of shear cracks. Applied Mathematics and Mechanics $(P M M) 28,1077-1087$.

Kostrov, B. V. (1966) Unsteady propagation of longitudinal shear cracks. Applied Mathematics and Mechanics $(P M M) 30,1241-11248$.

Loeber, J. F. and Sih, G. C. (1968) Diffraction of antiplane shear waves by a finite crack. J. Acoustical Society of America 44, 90-98.

Ma, C. C. and Chen, S. K. (1993) Exact transient analysis of an anti-plane semi-infinite crack subjected to dynamic body forces. Wave Motion 17, 161-171.

Ma, C. C. and Chen, S. K. (1994) Exact transient full-field analysis of an anti-plane subsurface crack subjected to dynamic impact loading. J. Appl. Mech. 61, 649-655.

Ma, C. C. and Hou, Y. C. (1990) Theoretical analysis of the transient response for a stationary inplane crack subjected to dynamic impact loading. Int. J. Engng Science 28, 1321-1329.

Ma, C. C. and Hou, Y. C. (1991) Transient analysis for antiplane crack subjected to dynamic loadings. J. Appl. Mech. 58, 703-709.

Noble, B. (1958) The Wiener-Hopf Technique, Pergamon Press.

Sih, G. C. and Embley, G. T. (1972) Impact response of a finite crack in plane extension. Int. J. Solids Structures 8, 977-993.

Sih, G. C. and Loeber, J. F. (1968) Torsional vibration of an elastic solid containing a penny-shaped crack. The Journal of the Acoustical Society of America 44, 1237-1245.

Sih, G. C. and Loeber, J. F. (1969) Wave propagation in an elastic solid with a line of discontinuity or finite crack. Quarterly of Applied Mathematics 27, 193-213.

Thau, S. A. and Lu, T. H. (1971) Transient stress intensity factor for a finite crack in an elastic solid caused by a dilatational wave. Int. J. Solids Structures 7, 731-750.

Tsai, C. H. and Ma, C. C. (1992) Transient analysis of a semi-infinite crack subjected to a dynamic concentrated forces. J. Appl. Mech. 59, 804-811.

Tsai, C. H. and Ma, C. C. (1993) The stress intensity factor of a subsurface inclined crack subjected to dynamic impact loading. Int. J. Solids Structures 30, 2163-2175. 